\title{
Design and Test of Resonators Using PiezoMUMPS Technology
}

\author{
Joan Pons-Nin*, Sergi Gorreta and \\ Manuel Domínguez
}

Micro and Nano Technologies Group, Electronic Engineering Department, Technical University of Catalonia, Barcelona, Spain.

*e-mail: joan.pons@upc.edu, Phone \#: 03493 4017475, Fax \#: 034934016756

\author{
Elena Blokhina, Diarmuid O’Connell \\ and Orla Feely
}

School of Electrical, Electronic and Communications Engineering, University College Dublin, Ireland.

\section{Suggested presentation format: Poster}

Keywords: Piezoelectric MEMS, MEMS resonators, mechanical modes.

\begin{abstract}
\section{Introduction}

This paper presents the first results obtained with devices fabricated on the inaugural run of the new PiezoMUMPS process [1]. This process has been introduced to the market in Autumn 2013 by MEMSCAP INC. as a response to the increasing research and commercial interest in piezoelectric MEMS devices. Using the well-known SOIMUMPS process as the baseline, this new standard technology offers a reliable, low cost and low temperature process that allows deposition and patterning of a 0.5 um Aluminum Nitride (AlN) active piezoelectric film on top of a Silicon-oninsulator (SOI) layer.

In our case, this technology was used to design, fabricate and test a number of structures that are intended for future use in several different research purposes, including resonators for sensing applications based on Pulsed-Digital-Oscillators (PDO) [2-4], low-cost power efficient resonant sensors and devices for energy harvesting [5,6]. Since the expected uses of these devices is mainly in oscillation-based applications, in this paper we focus on the analysis and comparison of the expected mechanical vibration modes and those excited and observed experimentally.
\end{abstract}

\section{Device design and simulation results}

The PiezoMUMPS process starts with a SOI wafer, which consists of a stack of, from bottom to top, handle wafer $(400 \mathrm{um})$, buried oxide $(1 \mathrm{um})$ and SOI device layer (10 um). Using a photolithography step on each side of the wafer, the SOI-device and handle wafer layers can be patterned and etched down to the buried oxide. Two metals layers, one for bond pads and another one for piezoelectric layer contacts, plus and an isolation oxide layer are also included in the process. The minimum feature size is $2 \mathrm{um}$. Let us also remark that this process allows fabrication of devices with full-thickness (400 um) suspended structures.

The set of devices designed includes rectangular, triangular and trapezoidal cantilevers, cantilevers with suspended (full-thickness) masses, bridges and membranes, all of them with various sizes and piezo-layer shapes. A microscope photograph of a die with the complete set of devices designed is shown in Figure 1. As an example, Figure 2 shows a close-view of three rectangular cantilevers of 100 um wide x 1200 um long x 10 um thick and with (full-thickness) proof masses at the tip ends. The AlN piezo layer is deposited on top of the whole area of the two outer cantilevers, and as two inter-digitized areas for the one in the middle. Note also that SOI-hole and trench features are used to electrically isolate neighboring devices.

Among others, one of the key features of PDO-based structures is their ability to selectively excite different mechanical vibration modes of the MEMS resonator. This is easily done in practice through real-time reconfiguration of the digital loop parameters of the circuit [3,4]. Thus, a first aim was to check what vibration modes could be actuated with the new PiezoMUMPS devices. Moreover, the suitability of the process for the design of piezoelectric vibration energy harvesters was validated by 
investigating the strain formulation of rectangular, trapezoidal and triangular cantilevers. Similar approaches have been used in the past to provide the theoretical principles of device geometry design, namely the use of trapezoidal and triangular cantilevers to improve the strain distribution across the material and thus generate more voltage [7,8]. In addition, mixed length cantilevers with fixed-mass proof masses have been designed to achieve lower frequency devices. Here again, our first aim was to test structures to check what vibration modes could be actuated.

According to these purposes, numerical simulations have been carried out using COMSOL and Coventor finite-element method (FEM) software packages. Such simulations included mechanical eigen modes simulations, time-independent (steady-state) and time-dependent (non-stationary) analysis of the devices. As an example, Figure 3 shows the deformation profiles corresponding to the first mechanical vibration mode for rectangular, triangular and rectangular with proof mass cantilever structures obtained from the eigen mode FEM analysis.

\section{Experimental results and discussion}

A first test was performed in order to check that electrical isolations between different devices and between different active layers worked fairly. Next and according to their abovementioned use as resonators for various applications, we used a probe station and an Agilent precision impedance analyzer to extensively check the mechanical vibration frequencies of the devices. All these measurements were made at low pressure (around $10 \mathrm{mbar}$ ) and room conditions of temperature and humidity. Fair oscillation waveforms and good quality factors have been obtained for most devices. As an example, Figure 4 shows experimental plots of module and phase around the first three resonant frequencies detected in a rectangular cantilever, marked as 'CC5' in Table 1.

The experimental results have been compared with the ones obtained previously from modal analysis using FEM simulations. Table 1 compares these results for the frequencies of the first vibration modes of several devices of different shapes. Let us remark that a nice matching between the simulated resonant frequencies and the experimental ones was found in most cases.

The cells in Table 1 marked with " $X$ " denote either resonance modes that were not found in the experiments or resonant behavior of poor quality. Based on a large number of FEM simulations, we have established that not all possible mechanical eigenmodes can be actuated in these devices. Since these devices operate in 31 mode, it is easier to actuate out-of-plane "bending" type of mechanical modes (as shown in fig. 3 and fig. 5a) than eigemodes involving rotation and torque of the resonators (fig. 5c). Therefore, many of "rotation" vibration modes are not visible. We also note that it is impossible to actuate in-plane bending mode (as shown in fig. 5b) since there is no transfer of displacement in in-plane direction.

\section{References}

[1] http://www.memscap.com/products/mumps/piezomumps

[2] J. Ricart, J. Pons, M. Domínguez, A. Rodríguez, E. Figueras, M.C. Horrillo, J. Gutiérrez, I. Sayago, 'Application of Pulsed Digital Oscillators to Volatile Organic Compounds Sensing'. Sensors and Actuators B: Chemical, Vol. 134-2, p. 773-779 (2008).

[3] E. Blokhina, J. Pons, J. Ricart, O. Feely, M. Domínguez, 'Control of MEMS vibration modes with Pulsed Digital Oscillators: Part I - Theory’. IEEE Trans. on Circuits and Systems I, vol.57-8, p. 1865-1878 (2010).

[4] J. Ricart, J. Pons, E. Blokhina, S. Gorreta, J. Hernando, T. Manzaneque, J.L. Sanchez-Rojas, O. Feely, M. Domínguez, 'Control of MEMS vibration modes with Pulsed Digital Oscillators: Part II - Simulation and experimental results'. IEEE Trans. on Circuits and Systems I, vol.57-8, p. 1879-1890 (2010).

[5] D. Shen, J. H. Park, J. H. Noh, S. Y. Choe, S. H. Kim, H. C. Wikle III, D. J. Kim,' Micromachined PZT cantilever based on SOI structure for low frequency vibration energy harvesting', Sensors and Actuators A, Vol. 154, p. 103-108 (2009).

[6] M. Marzencki, Y. Ammar and S. Basrour, 'Design, fabrication and characterization of a piezoelectric microgenerator including a power management circuit', Proc. of DTIP of MEMS and MOEMS, p. 1-4, Stresa, Italy, 25-27 April 2007 (2007).

[7] Z. S. Chen, Y. M. Yang and G. Q. Deng, Proc. of Conf. Sustainable Power Generation and Supply '09, p. 16, Nanjing, China, 6-7 April 2009. 
[8] S. Roundy et al., 'Improving Power Output for Vibration Based Energy Scavengers', IEEE Pervasive Computing , p. 28-36 (2005).

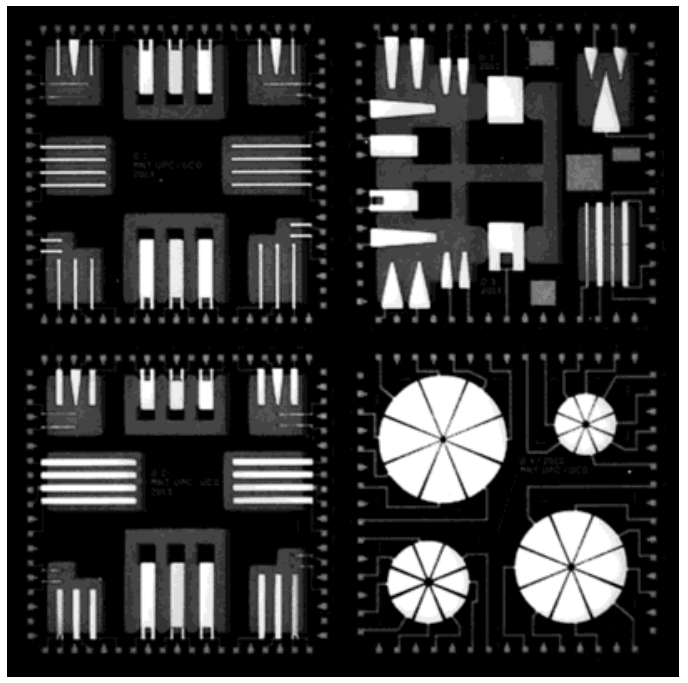

Figure 1. Photograph of a PiezoMUMPS die.

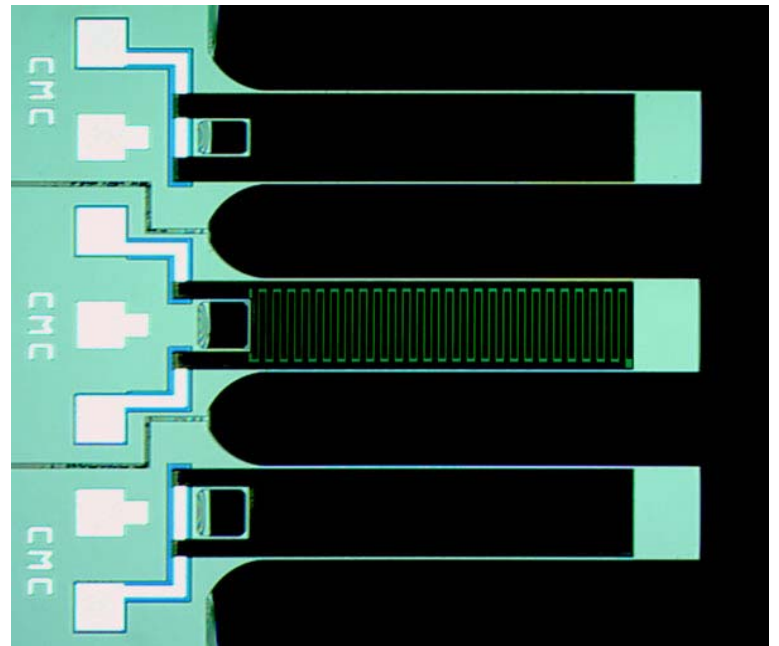

Figure 2. Photograph of a set of cantilevers with proof masses at their tips.

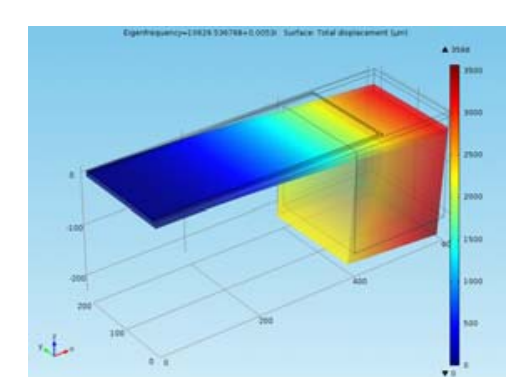

Figure 3. FEM simulation results showing geometric deformations corresponding to the first vibration mode of different cantilever structures.
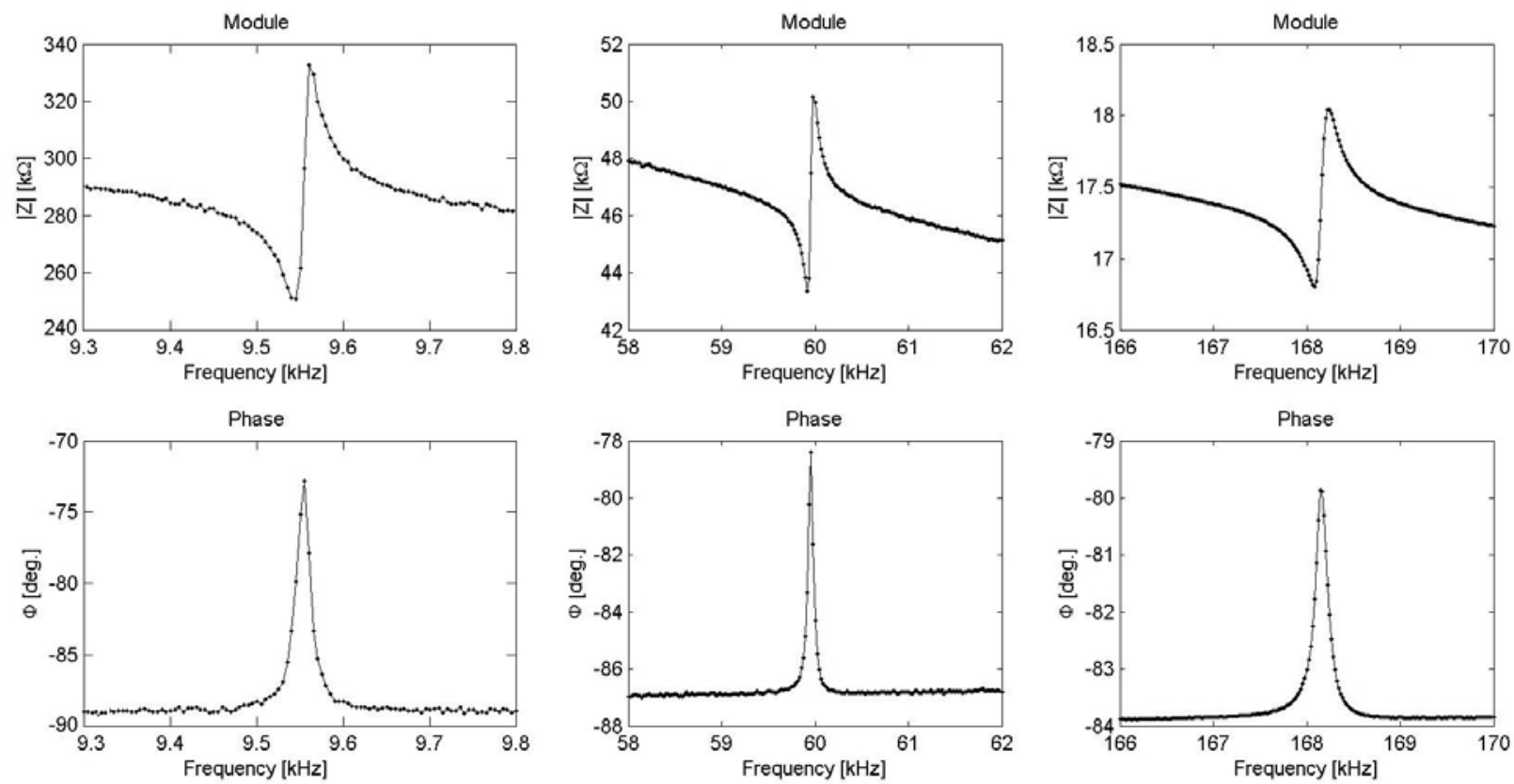

Figure 4. Module and phase of the first three resonances detected with cantilever CC5. 


\begin{tabular}{|c|c|r|c|c|c|c|}
\hline Device & Cantilever shape & Data origin & $1^{\text {st }}$ mode & $2^{\text {nd }}$ mode & $3^{\text {rd }}$ mode & $4^{\text {th }}$ mode \\
\hline \multirow{2}{*}{ CB4 } & \multirow{2}{*}{ Rectangular } & FEM sims. & 20.7 & 95.2 & 130 & 346 \\
& & Experimental & 21.4 & $\mathrm{X}$ & 134.2 & 375 \\
\hline \multirow{2}{*}{ CC5 } & \multirow{2}{*}{ Rectangular } & FEM sims. & 9.26 & 58.02 & 80.36 & 162.5 \\
& & Experimental & 9.55 & 60 & $\mathrm{X}$ & 168 \\
\hline \multirow{2}{*}{ CMC } & \multirow{2}{*}{ Rectangular } & FEM sims. & 2.81 & 8.98 & 17.3 & 93.1 \\
& with proof mass & Experimental & 2.86 & $\mathrm{X}$ & 17.2 & 112.7 \\
\hline \multirow{2}{*}{ ZC } & \multirow{2}{*}{ Trapezoidal } & FEM sims. & 53 & 287 & 456 & 770 \\
& & Experimental & 46.2 & 250 & $\mathrm{X}$ & $\mathrm{X}$ \\
\hline \multirow{2}{*}{ TC } & \multirow{2}{*}{ Triangular } & FEM sims. & 49.4 & 213 & 352 & 517 \\
& & Experimental & 44 & 190.5 & $\mathrm{X}$ & 462 \\
\hline
\end{tabular}

Table 1. Comparison of simulation results with experimental data for the first four frequency modes in several devices. All frequency values are in $\mathrm{kHz}$.

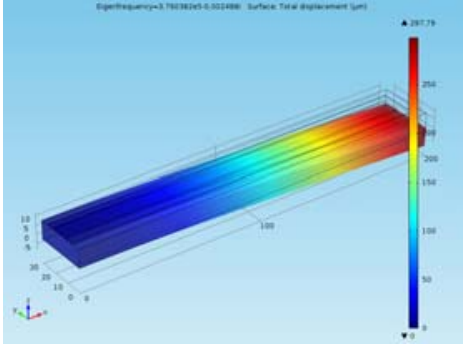

a

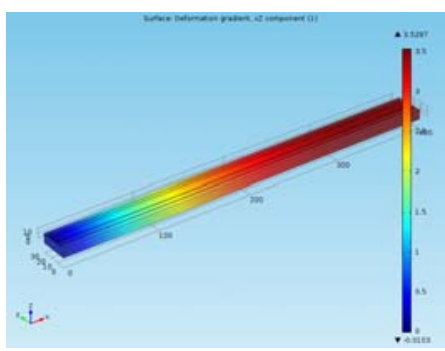

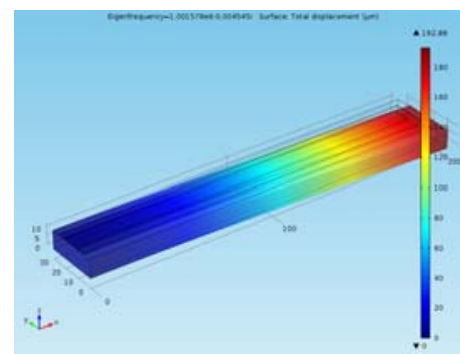

$\mathrm{b}$

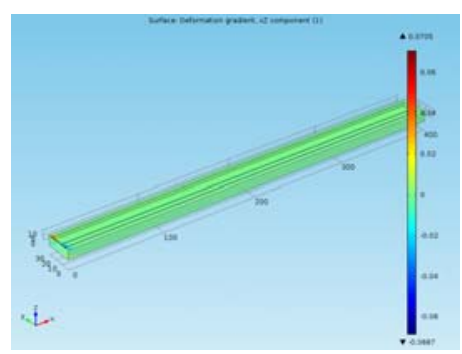

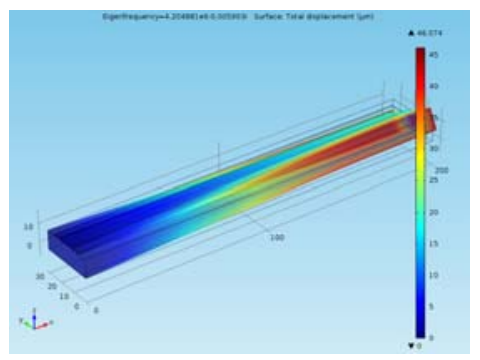

C

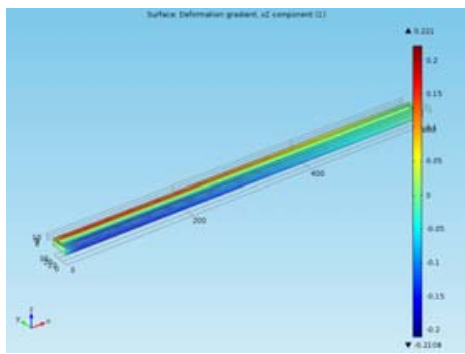

Figure 5. Three different types of mechnical eigemodes: their shapes (upper row) and the tensor XZ component of deformation gradient (lower row). There are two types on bending modes (out-of-plane XZ and in-plane XY bending) and rotation/torque modes.

\section{Short biography}

Joan Pons-Nin is an Associate Professor with the Department of Electronic Engineering at the Telecommunications Engineering School of Barcelona, Spain. He received the MSc (1989) and his Ph.D (1995) degrees in Electronic Engineering from the Universitat Politècnica de Catalunya (UPC). He is currently working on oscillators for MEMS resonators and MEMS reliability. 